\title{
A Three-Level Approach for Analyzing User Behavior in Ongoing Relationships
}

\author{
Enric Mor $^{1}$, Muriel Garreta-Domingo ${ }^{2}$, Julià Minguillón ${ }^{1}$, Sheena Lewis ${ }^{3}$ \\ ${ }^{1}$ Computer Science, Multimedia and Telecommunication Dept - Universitat Oberta de \\ Catalunya - Rambla del Poblenou 156, 08018 Barcelona, Spain \\ \{emor ,jminguillona\}@uoc.edu \\ ${ }^{2}$ Learning Technologies Dept - Universitat Oberta de Catalunya - \\ Av. Tibidabo 39, 08035 Barcelona, Spain \\ murielgd@uoc.edu \\ ${ }^{3}$ College of Computing - Georgia Institute of Technology - \\ $855^{\text {th }}$ Street Atlanta, GA, USA 30318 \\ sheena@gatech.edu
}

\begin{abstract}
This paper describes a hybrid methodology to study users in ongoing relationships based on three levels of user data analysis. Most user-centered design methods are ideal for the analysis of users' needs, wants, and expectations at a specific point in time. However, nowadays, most online applications and services have recurrent users whose characteristics might vary not only over time but also depending on the task they want to accomplish and the context in which they are accomplishing it. Therefore, the common user research methods are not adequate for providing long term feedback. Our threelevel approach methodology combines qualitative and quantitative data for analyzing user behavior over an extended period of time. The present study is based on an e-learning environment, which is a great example of a website with recurrent users whose behavior changes over time.
\end{abstract}

Keywords: Long-term human-computer interaction, ongoing relationships, log analysis, combining methodologies, user behavior, virtual learning environments, e-learning.

\section{Introduction}

Analyzing the user experience of a website is a crucial element for satisfying users and offering them what they need and expect. There are several user-centered design methods that provide this analysis; however, none of the most common methods are suitable to study the behavior of recurrent users. Yet nowadays most websites have recurrent users that behave differently as they gain experience, create habits, and construct mental models that modify their behavior over time. 
In this paper, we define a new hybrid methodology that combines some of these methods together with usage data and log analysis. Initially, it has been developed and used in order to study users in an e-learning environment. However, it is useful for other online applications where users log in to seek information and to develop different tasks over time such as corporate intranets, on-line banking or e-commerce sites. The three levels of analysis of the proposed methodology are based on the study of user behavior during different time periods, and therefore, with users trying to accomplish different goals. Hence, the analysis levels are classified depending on the usage data collected [9], the time span taken into account - short term, mid term and long term - and the user's goal.

This three-level approach helps designers understand the behavior and attitude of users both at a specific point of time and over different time periods, as well as how mixing usage data with common user research methods provides richer and more specific information about users' behavior and system usage than using these methods independently. In the case of virtual e-learning environments, this approach allows an accurate analysis and gives a complete view of system usage and student behavior because at each level the user's goals can be learned and correlated with the data analysis. As mentioned earlier, this three level method can be also applied in other interactive environments providing good results and information about users' behavior and system

This paper is organized as follows: Section 2 describes the importance of long term user-centered design and an overview of the existing research related to our framework. Section 3 describes a three-level framework for study user behavior in virtual environments. Section 4 presents the application of the three-level framework in the UOC virtual campus environment. Finally, the conclusions drawn from this research and future research are summarized in Section 5.

\section{Long term user-centered design and related work}

Most websites are built with the intention of providing a good user experience, which will encourage users to return. In fact, a website that provides users with desired services as well as a good design may flourish for months, even years. Clearly, evaluations must be conducted in order for designers to determine user satisfaction. In fact, most companies use traditional summative evaluation methods. However, these types of evaluation methods provide data from users' initial reaction to the website (i.e. information at a single point in time). Yet, users' experiences change over continued use of a website because users become accustomed to the system, which may lead for them to behave differently than during their initial visits. Analyzing this change would help designers understand the changes in user needs, identify usability problems, and recognize ways to improve the user experience as well as how to keep them engaged. Therefore, designers should not only consider performing traditional user evaluations after initial deployment but also applying user-centered design (UCD) techniques over an extended period of time.

Performing long-term UCD methods is not a new concept. In fact, long-term UCD methods have been used to identify the transition from novice to expert users on 
systems after deployment $[19,20]$. Understanding the transitions between novices and experts is important since many times if novices do not transition to experts, they may become frustrated and less likely to continue to use the system. With this information, systems can be built to transition novices to experts in a shorter period of time [19]. Much of the research that has been performed in this area has focused on cognition $[15,19,4]$ without considering user intentions with the system. Additional research performed identifies experts in the laboratory settings performing specific tasks [6]. Nevertheless, using predefined tasks does not allow data to be gathered about tasks performed in the real setting, which are aligned with the users' overall goals.

Clearly, log file analysis affords evaluation of users in the real setting after deployment in order to provide understanding of user navigation and real interface usage data [2]. Many studies have concluded that knowing user behavior can positively increase the effectiveness of web design [12]. Additionally, the methods in how to extract information from users into logs files have been extensively been explored [9, 22, 11]. However, log analysis provides quantitative results, which can be significantly enriched by including qualitative data during analysis. Thus, we propose a combined methodology in order to provide a more enlightening understanding of the user behavior as well as design implications in order to improve the user experience.

Other research uses remote user testing to gather data after deployment in a realistic setting. Providing self-reporting tools to allow users to identify critical incidents during normal system use can help make usability improvements [8]. Additionally, remote user testing has been used to create logs in order to view user behavior and patterns [16]. Yet, our study differs in that we aim to merge quantitative and qualitative methods to view how the user achieves his/her goals in order to improve the user experience as well as usability.

\section{Three-level user analysis framework}

Our proposal is based on a three-level framework for user behavior analysis with an emphasis on navigation paths. Three different user navigation and behavior patterns levels are usually distinguished, namely short term, medium term, and long term. From a navigation and HCI perspective, these levels are deemed as session level, activity level, and wish level, respectively. Our focus is on the users' knowledge and how to model their successful navigation behavior paths for each of these levels. The analysis of the user behavior at each level provides relevant information for constructing a user model and allows gathering three levels of information that can be utilized to gain insight about the users and evaluate system usage, and therefore improve the system. At each level, the quantitative information obtained is combined with qualitative information gathered from UCD methods [7].

The three-level user analysis framework is a long-term UCD methodology that decomposes user analysis in different periods of time. This decomposition provides practically constant feedback on user behavior while also enriching analysis over time. This analysis technique can also complement more traditional methods that provide information of a point in time. 
Within the context of e-learning, in which we have tested our framework, the methodology provides information about what students actually do in virtual environments. Our methodology is hybrid in two ways: 1) it merges qualitative and quantitative data and 2) it combines navigation and log analysis data with the user's "external” data. By external in the context of e-learning, we mean socio-demographic and academic data. Conversely, in an e-commerce environment, for example, the external data would be socio-demographic and purchase data.

Although our methodology can be extrapolated to any websites with recurrent and registered users, it needs to be adapted to the specificities of each context. In our case, in order to analyze e-learning environments, the three levels are session level, academic course level and lifelong learning level. These three levels arise in a natural and hierarchical way from the use that the students make of the virtual environment and the distinctive dynamics of their learning and training activities when carrying out online distance studies. That is, the session level captures the way users navigate with particular goals in mind and it considers each student log into the virtual learning environment. The mid term level is the academic semester level and takes into account the single user sessions in a continuous flow during the course duration; this typically ranging from a few weeks to a few months. The long term level takes into account how students evolve from the beginning of a degree until they successfully finish it; this level can span from a few semesters to several years.

In a virtual learning environment, knowing what users do and how they navigate is even more important than knowing how they should navigate and which actions they should perform. Therefore, at each level of study, user navigation and behavior must be regarded in relation with the navigation and behavior models used when the elearning environment and the courses were designed. Thus, the virtual learning environment can be measured and new, unexpected facts can be discovered.

\subsection{Short term level}

The first level is called session level, the shortest period of time of the three levels. There are two main expected results from the short term level analysis. The first result is a system usage analysis by means of user navigation paths and a grouping proposal of these paths into patterns. The second is the information from combining the navigation patterns with qualitative and contextual data.

The data collected and used in the session level is the lowest unit of information, which can be utilized for analysis as a standard log file. When users navigate through an interactive web environment, their selections are recorded, which can be later analyzed for modeling purposes [13, 9]. Most of this information is collected by the web servers in the form of server log files, usually according to the Apache Common Log Format. This includes the actions performed, the information accessed, and the path users traversed to arrive at certain points. Since the standard log files do not provide enough data [3], our approach includes a marking strategy in order to capture accurately the user navigation paths. This strategy has been previously described in [18] and differentiates action based and content based marks.

The analysis performed in this short term level is oriented to obtain user navigation paths and patterns. These navigation paths demonstrate what users really do in the 
virtual environment but not what they intended to do. Hence, the navigation patterns are combined with qualitative data obtained from UCD methods, especially inquiry methods such as interviews, focus groups, and surveys. Moreover, inspection methods such as cognitive walkthroughs are also used. On the other hand, there also goals that we can assume and that provide qualitative data that can be combined with navigation patterns. At this level, this data is very useful for the analysis of usability problems. Therefore, the user behavior and navigation data obtained in this level can be used to evaluate and improve the usability $[9,11]$ of the system. As a complement to the qualitative data and the goals that can be assumed by the system designers, we can gather specific information on the user's goal and context by directly asking the user after logging in. It is important to note that this technique can not be used too persistently but instead should be used as a confirmation tool. Results of this level are discussed in the next section and in [18].

\subsection{Mid term level}

The second level is the activity level. The time span of this level depends on the interactive environment or the application being analyzed. For example, in an elearning context, the activity level is the analysis of the user's progress during a course. In the case of a banking website, the activity level can be a monthly period, from one pay check to the next.

At this level, we do not ask for the user's goal but instead, we assume the goal. For instance, we assume that most students want to learn the course contents in order to pass the course evaluation activities. The activity level is obtained from the addition of all session data during a specified amount of time. The goal and context defined by the user at each session is used to classify sessions and define patterns of users' behavior. This second level informs designers and developers about the user experience and how the user's goals correlate with the stakeholders' goals.

\subsection{Long term level}

The wish level is the third level, and the user's goal here is to accomplish the task they had in mind when he or she initiated the relationship. In an e-learning environment, the goal is both learning and obtaining a degree. The wish level considers the evolution of activity levels data and classifies them according to the goal and the result obtained by the user. This last level of analysis is important in order to learn about the long term user experience and users' fidelity in the sense of a lifelong relationship with the institution.

As previously mentioned, in an e-learning environment, those three levels are concretized as session level, academic course level and lifelong learning level. The information obtained at the session level is used to increase the system's efficiency and learn how students navigate in the site and how it correlates to their stated goal. At the course level, the data is essential from an educational point of view: it is used to match the instructional issues and the course itineraries with the student behavior. With the lifelong learning level, we analyze how students evolve from the beginning 
of their studies and we obtain successful paths between courses. In turn, this information is used to decrease student dropout and to improve the student learning experience.

Studying the user activity using this three level method in a virtual e-learning environment allows an accurate approach and gives a complete view of system usage and student behavior because at each level the user's goals can be determined and correlated with the data analysis.

\section{Our context: UOC's student behavior}

The Universitat Oberta de Catalunya (UOC, in English known as Open University of Catalonia) is a completely online university which offers 20 official degrees, several graduate programs and post-graduate studies, and a doctoral degree, with more than 40,000 students and more than 2,500 people, including instructional designers, teachers, tutors, academic and technical staff. The UOC virtual campus is an integrated e-learning environment, which allows users to communicate with each other using a mail system with complete timetable independence, using an asynchronous approach. Other features of the virtual campus include an personal schedule, a news service, virtual classrooms and laboratories, a digital library and other e-learning related tools. UOC has a student centered pedagogical model that ensures a guided learning path through the use of selected learning resources, according to the experience of a team composed of instructional designers, usability experts, and teachers. In addition to this student centered pedagogical model, a learner centered design perspective is used to design and evaluate the virtual campus environment and tools.

Within the UOC virtual campus, each subject has a virtual classroom with all the needed elements for the development of the teaching and learning process: e-mail, access to documentation, the activity based teaching plan, access to evaluation results, access to the teacher board, forums, debates, etc. The virtual campus classrooms are the meeting point of the different learning activities. The teaching plan is a document which summarizes all the learning activities the students must carry out in order to follow the proposed learning path, helping them to achieve the learning goals and competences developed by each subject.

In any virtual learning environment, such as the UOC virtual campus, the student behavior can be collected and analyzed using web mining techniques [5]. The data analyzed will be very useful for following the student behavior and for adjusting the formative proposal to students' needs. In order to offer learning paths according to the student behavior, these types of studies are proposed as basic tools for capturing the real intentions of learners in the virtual campus. For instance, it is very important to know the actual enrollment behavior of students in the Computer Engineering degree (a two year program) in order to redesign the requirements and the recommendations given to students. Similarly, dropout is also an important issue which may be better understood if relevant variables are identified, such as undesirable combinations of difficult courses, which may be the cause of frustration and eventually withdrawal from the course or university. 
In order to show the possibilities of applying the proposed methodology to the particular case of the UOC virtual campus, we describe several experiments designed to capture user behavior and to find relationships between user navigational behavior and user experience in a virtual learning environment. This list of possible experiments is by no means exhaustive. It is only a starting point for analyzing user behavior in virtual learning environments and the possible relationships with other key aspects of user experience and satisfaction, which are strongly related to academic performance and dropout, two very important issues.

\subsection{Short term level experiments}

In this level, a simple experiment has been designed to study user behavior when logging into the virtual campus and navigating between the different sections available from the initial starting page. Basically, when a student logs into the virtual campus, he or she has access to several areas: the mailbox (denoted MAIL), the list of their current courses (LIST), the discussion boards for each course (BOARDS), the virtual classroom space (CLASSROOM), other secondary areas including the digital library (OTHER), and simply exiting the virtual campus (EXIT). We are interested in analyzing how students' behavior evolves along the academic semester, in order to determine whether the initial page should be adapted to each student's profile or not. Actually, the starting page is often redesigned to accommodate users' preferences, according to the results of qualitative studies using focus groups and other techniques. Nevertheless, it is interesting to understand real users' behavior because the initial web page could be automatically adapted to capture the expected behavior according to special dates (e.g. at the beginning or the end of the academic semester).

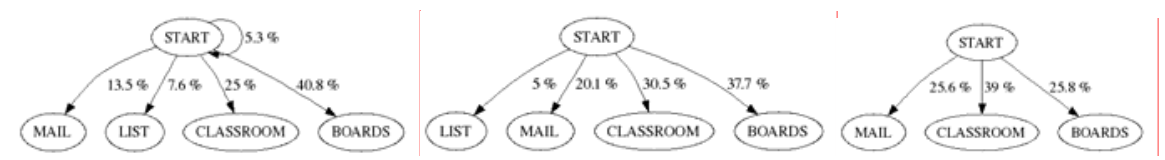

Figure 1. From left to right, first action taken by students from the initial web page the first day of the semester, the day before the face-to-face meeting and the day before the end of the semester.

Figure 1 shows the initial action taken by students once they log into the virtual campus for three different days, selected because of their importance. The first day of the semester, students are expected to go to the boards to read the welcome messages and initial directions given by teachers, while the mailbox is still not used at its possible potential as a communication tool. The mailbox is more and more accessed as students evolve along the academic semester, while message boards follow the opposite behavior, as expected. Notice that at the beginning of the academic semester, a few students (5.3\%) refresh the initial page, seemingly because they are waiting for the teacher to provide them with the appropriate directions (keeping in mind that it is an asynchronous communication model). On the other hand, LIST (a direct access to 
the list of subjects) is no longer accessed the last day of the academic semester, as expected since students have already completed their required activities.

This experiment shows that all the "expected" behavior can be really captured and integrated into the virtual campus. The figures are statistically significant, as 14,616, 8,732 and 9,641 different students (in the Catalan virtual campus, enrolled into any of the official degrees) have been uniquely identified. Thus, the virtual campus is a potential user behavior laboratory that can be used to study many different aspects related to the learning process and the design of the virtual learning environment both from a pedagogical point of view and from a UCD point of view.

\subsection{Mid term level experiments}

Another interesting experiment, described in [1], shows the use of web mining techniques for predicting user behavior with respect to the relationship between the publication of a proposed exercise and the navigational patterns during the period available for a solution. In this case, our main goal was to analyze such user navigational behavior for extracting information that could be used to validate several aspects related to virtual campus design and usability but also to determine the optimal scheduling for each course depending on user profile. We intend to extend the sequencing capabilities of standard learning management systems to include the concept of recommended itinerary, by combining teachers' expertise with learned experience acquired by virtual campus usage analysis, in order to incorporate personalization capabilities into the virtual campus [17], and making the virtual campus a truly intelligent tutoring system.

\subsection{Long term level experiments}

Finally, in the long term level, it is important to analyze the decisions taken by students with respect to their learning path and creating their own curricula, in order to see whether all the recommendations given by teachers, tutors, and administration are truly understood and properly used. In order to analyze the students' behavior at this level, we have carried out a simple experiment which shows the reality regarding how students enroll into the offered subjects at each academic semester, showing the differences between the official and the real learning pace for the Computer Engineering degree. Table 1 shows the number of students enrolled into mandatory subjects each semester according to the academic semester each subject is supposed to be done. This table has been generated using accumulated data from ten academic semesters, since Spring 2001 to Fall 2005.

In the first semester, students are supposed to enroll into four mandatory subjects and a few optional subjects, while a simple statistical analysis reveals that most students only take one or two subjects each semester. Among the vast amount of available subjects (the four proposed but also other subjects which can be taken as credits needed to fulfill the degree requirements), most students chose them from a limited subset, the four supposed to be taken and a few courses very different in nature; for example, one optional course in Computer Graphics and other optional 
Database course from the offer from the second semester. This fact reveals that some students have already acquired the basic competences in the technical degree and can advance faster in the Database specialization. Two subjects, one in Object Oriented Software Engineering (11) and other in Artificial Intelligence I (14) are the most popular, while other subject known to be difficult, Computer Architecture (13), is not likely to be chosen in the first semester.

\begin{tabular}{|c|c|c|c|c|c|c|c|c|c|c|c|c|c|c|}
\hline & \multicolumn{9}{|c|}{ 1st sem. } & \multicolumn{4}{c|}{ 2nd sem. } & \multicolumn{3}{c|}{ 3rd sem. } & 4th sem. & \\
\hline Sem. & 11 & 12 & 13 & 14 & 21 & 22 & 23 & 24 & 31 & 32 & 33 & 41 & 42 & Total \\
\hline 1 & $\mathbf{4 7 2}$ & $\mathbf{1 7 8}$ & 77 & $\mathbf{3 8 9}$ & 7 & 31 & 8 & 5 & 0 & 2 & 61 & 1 & 0 & 785 \\
\hline 2 & $\mathbf{1 0 4}$ & $\mathbf{1 2 0}$ & $\mathbf{1 5 0}$ & $\mathbf{1 1 7}$ & $\mathbf{1 1 7}$ & $\mathbf{8 1}$ & 33 & 64 & 10 & 10 & $\mathbf{7 6}$ & 12 & 0 & 576 \\
\hline 3 & 25 & $\mathbf{1 4 5}$ & 59 & 37 & $\mathbf{1 6 9}$ & 67 & $\mathbf{8 1}$ & 49 & 31 & 11 & $\mathbf{9 3}$ & 10 & 1 & 396 \\
\hline 4 & 14 & 50 & 35 & 22 & $\mathbf{6 6}$ & $\mathbf{8 0}$ & $\mathbf{6 1}$ & $\mathbf{8 0}$ & $\mathbf{8 4}$ & 40 & $\mathbf{6 6}$ & 29 & 3 & 300 \\
\hline 5 & 4 & 31 & 21 & 10 & 39 & 36 & 35 & $\mathbf{6 7}$ & 43 & $\mathbf{5 4}$ & 45 & $\mathbf{5 9}$ & 15 & 241 \\
\hline 6 & 0 & 17 & 8 & 8 & 19 & $\mathbf{3 3}$ & 26 & 31 & 19 & $\mathbf{3 4}$ & $\mathbf{3 3}$ & $\mathbf{3 8}$ & 23 & 170 \\
\hline
\end{tabular}

Table 1. Enrolment by semester according to each academic semester.

Other interesting fact is that students do not follow exactly the given recommendations for taking the courses. For example, Artificial Intelligence II (24) is supposed to be done after Artificial Intelligence I (14), but only 64 out of 389 possible students do it during the first and second semesters, while Compilers I (33) has been done by 230 students by the third semester but only 52 have enrolled into Compilers II (41) by the fourth one. The main reason is that students prefer to finish complete semesters instead of following the proposed paths for specialization. Another reason is that not all students pass the evaluation procedure and must retake course.

\section{Discussion and conclusions}

Knowing what users do in a virtual environment provides invaluable data to their designers. Traditional user-centered design methods provide the knowledge of a point in time, but updating the knowledge over an extended period of time is as valuable as the short term information. User behavior depends on the user type, the users' goals, and the evolution of their relationship with the environment. Our three-level framework provides a methodology to clearly study this change.

Our experiments within the context of UOC show that it is possible to identify different paths depending on the time period of the analysis. Therefore, designers can use this information to improve the usefulness of the system, and consequently, the overall user experience.

In future studies, we plan to continue to perform more experiments, especially for the mid term and long term levels, for which we have gathered less data until now. Additionally, as we collect more data, we also plan to continue to combine both qualitative and quantitative data to do the analysis of each level.

Acknowledgments. This work has been partially supported by a Spanish government grant under the project PERSONAL (TIN2006-15107-C02-01). 


\section{References}

1. Carbó, J. M., Mor, E., Minguillón, J.: User Navigational Behavior in e-Learning Virtual Environments. In Proceedings of the ACM International Conference on Web Intelligence. (2005) 243-249

2. Catledge, L. D., \& Pitkow, J. E.: Characterizing Browsing Strategies in the World-Wide Web. (1995) 1065-1073

3. Cooley, R., Mobasher, B., Srivastava, J.: Data Preparation for Mining World Wide Web Browsing Patterns. Knowledge and Information Systems, Vol. 1 num. 1 (1999) 5-32

4. Dreyfus, Stuart E., Dreyfus, Hubert L. A Five-Stage Model of the Mental Activities Involved in Directed Skill Acquisition. (1980) University of California, Berkley

5. El-Ramly, M., Stroulia, E.: Analysis of web-usage behavior for focused web sites: a case study. Journal of Software Maintenance and Evolution: Research and Practice, vol. 16(1-2) (2004) 129-150

6. Ericsson, K.A., Lehmann, A.C. Expert and Exceptional Performance: Evidence of Maximal Adaption to Task Constraints. Annual Review of Psychology, Vol. 47, 1996.

7. Hackos, J. T., \& Redish, J. C.: User and task analysis for interface design. John Wiley \& Sons, Inc, New York, NY, USA (1998)

8. Hartson, H. R. \& Castillo, J. C. Remote evaluation for post-deployment usability improvement. In Proceedings of the Working Conference on Advanced Visual interfaces (L'Aquila, Italy, May 24 - 27, 1998 AVI '98. ACM Press, New York, NY, 22-29.

9. Hilbert, D. M., \& Redmiles, D. F.: Extracting Usability Information from User Interface Events. ACM Comput. Surv., 32 (2000) 384-421

10. Hübscher, R., \& Puntambekar, S.: Modeling Learners as Individuals and as Groups. Lecture Notes in Computer Science, 3137 (2004) 300-303

11. Ivory, M. Y., \& Hearst, M. A.: The State of the Art in Automating Usability Evaluation of User Interfaces. ACM Comput. Surv., 33 (2001) 470-516

12. Juvina, I., Trausan-Matu, S., Iosif, G. et al.: Analysis of Web Browsing Behavior - a Great Potential for Psychological Research. (2002) 170-178

13. Juvina, I., \& van Oostendorp, H.: Individual differences and behavioral aspects involved in modeling web navigation, Vol. 3196 (2004)

14. Kort, J., \& Poot, H. d.: Usage Analysis: Combining Logging and Qualitative Methods. (2005) 2121-2122

15. LaFrance, M. 1989. The quality of expertise: implications of expert-novice differences for knowledge acquisition. SIGART Bull., 108 (Apr 1989), 6-14.

16. Millen, D., (1999). Remote Usability Evaluation: User participation in the Design of a Web-Based email Service. SIGGROUP Bulletin, Vol. 20. No. 1.

17. Mor, E. \& Minguillón, J., E-learning personalization based on itineraries and long-term navigational behavior. Proceedings of the Thirteenth World Wide Web Conference, New York City, NY, volume 2 (2004) 264-265

18. Mor, E., Minguillón, J., Santanach, F. 2007. Capturing User Behavior in E-Learning Environments. In Proceedings of the 3rd International Conference Web Information Systems and Technologies. Barcelona, Spain.

19. Popovic, Vesna (2000) Expert ans Novice User Differences and Implications for Product Design Usability. In Proceedings IEA 2000/HFES 2000 Congress, pp. 933-936.

20. Prümper, J., Frese, M., Zapf, D., and Brodbeck, F. C. 1991. Errors in computerized office work: differences between novice and expert users. SIGCHI Bull. 23, 2 (Mar. 1991), 63-66.

21. Renaud, K., \& Gray, P.: Making Sense of Low-Level Usage Data to Understand User Activities. (2004) 115-124

22. Spiliopoulou, M.: Web Usage Mining for Web Site Evaluation. Commun ACM, 43 (2000) 127-134 